\title{
In vivo dark-field reflection-mode photoacoustic microscopy
}

\author{
Konstantin Maslov \\ Optical Imaging Laboratory, Department of Biomedical Engineering, Texas A\&M University, \\ 3120 TAMU, College Station, Texas 77843-3120 \\ Gheorghe Stoica \\ Department of Veterinary Pathobiology, Texas A\&M University, 5547 TAMU, College Station, Texas 77843-5547
}

Lihong V. Wang

Optical Imaging Laboratory, Department of Biomedical Engineering, Texas A\&M University, 3120 TAMU, College Station, Texas 77843-3120

Received October 4, 2004

Reflection-mode photoacoustic microscopy with dark-field laser pulse illumination and high-numericalaperture ultrasonic detection is designed and implemented in noninvasively imaged blood vessels in the skin in vivo. Dark-field optical illumination minimizes the interference caused by strong photoacoustic signals from superficial structures. A high-numerical-aperture acoustic lens provides high lateral resolution, $45-120 \mu \mathrm{m}$ in this system. A broadband ultrasonic detection system provides high axial resolution, estimated to be $\sim 15 \mu \mathrm{m}$. The optical illumination and ultrasonic detection are in a coaxial confocal configuration for optimal image quality. The system is capable of imaging optical-absorption contrast as deep as $3 \mathrm{~mm}$ in biological tissue. (C) 2005 Optical Society of America

OCIS codes: $170.3880,170.5120,180.5810$.

The ability to image the microvascular network in skin is invaluable in dermatology ${ }^{1}$ and related cancer research. ${ }^{2,3}$ A promising technique for accomplishing this objective is photoacoustic microscopy. A common goal of all imaging techniques is to achieve the highest possible resolution and contrast at the desired penetration depth within a reasonable time. Current high-resolution optical imaging techniques, such as confocal microscopy and optical coherence tomography, can image only approximately one transport mean free path $(1-2 \mathrm{~mm})$ into biological tissues because these techniques depend on ballistic or quasiballistic photons. These techniques are sensitive to the backscattering that is related to tissue morphology, but they are insensitive to the optical absorption that is related to important biochemical information. Photoacoustic imaging does not depend on ballistic or quasi-ballistic photons and can, therefore, penetrate deeper. Further, it provides high optical-absorption contrast while it maintains high ultrasonic resolution. Consequently, structures with high optical absorption coefficients, such as blood vessels, can be imaged clearly.

High image resolution was achieved previously with circular-scanning photoacoustic computed tomography. ${ }^{4,5}$ Although a full $360^{\circ}$ scan about an object provides high resolution and minimizes artifacts, it can be accomplished only with elevated objects, such as brain or breast. Previously investigated planar reflection-mode techniques ${ }^{6-8}$ are not limited by the shape of the sample, but they may suffer from the strong photoacoustic waves emitted from optical absorbers near the surface, such as hair follicles and melanin, whose acoustic reverberations can potentially overshadow the much weaker photoacoustic signals from structures deep in the tissue.

In this Letter we report a reflection-mode microscopic photoacoustic imaging technique that uses dark-field illumination, as in dark-field microscopy, to prevent the occurrence of such problems. To achieve high image resolution and high sensitivity we utilize a high-frequency, large-numerical-aperture (NA) spherically focused ultrasonic transducer that is coaxial and confocal with the optical illumination.

Because of the strong light scattering that occurs in biological tissue, photoacoustic image resolution beyond one optical transport mean free path into biological tissue is determined primarily by the ultrasonic detection parameters. To provide high resolution, the acoustic detector must have a wide bandwidth and a large NA. However, increasing the ultrasonic frequency too much can result in an undesirably small penetration depth because the ultrasonic attenuation in biological tissue, $0.7-3 \mathrm{~dB} /(\mathrm{cm} / \mathrm{MHz})$ for human skin, ${ }^{9}$ for example, increases linearly with the frequency. Therefore, a large NA is essential for the desired resolution.

Figure 1 shows the experimental setup for our photoacoustic microscopic system. A $Q$-switched pulsed Nd:YAG laser (Brilliant B, BigSky), operating at $532 \mathrm{~nm}$, delivers $300 \mathrm{~mJ}$ of energy per pulse to a

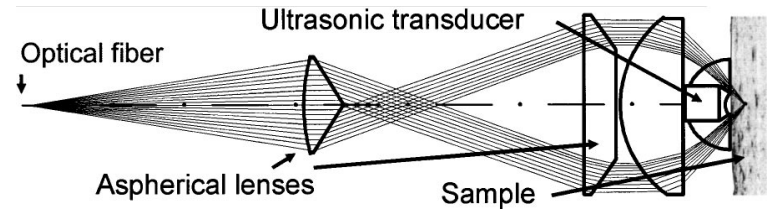

Fig. 1. Schematic of the photoacoustic sensor of the imaging system. 
0.60-mm-diameter optical fiber. The laser pulse width is $6.5 \mathrm{~ns}$, and the pulse repetition rate is $10 \mathrm{~Hz}$. The fiber output is coaxially positioned on a three-dimensional precision mechanical scanner with a focused ultrasonic transducer (Panametrics). The transducer has a center frequency of $50 \mathrm{MHz}$ and a nominal bandwidth of $70 \%$ and is attached to an inhouse-constructed concave lens (aperture diameter, $D=5.5 \mathrm{~mm}$; working distance, $5.6 \mathrm{~mm}$ ). This aperture provides a NA of 0.44 , which is considered relatively large in ultrasonics. The laser light from the fiber is expanded by a conical lens and then focused through an optical condenser with a NA of 1.1. The optical focal region overlaps the focal spot of the ultrasonic transducer, thus forming a confocal optical dark-field illumination and ultrasonic detection configuration.

Compared with alternative designs that involve bright-field illumination, this design provides the following advantages: First, a large illumination area reduces the optical fluence on the sample surface to less than $1 \mathrm{~mJ} / \mathrm{cm}^{2}$, which is well within the safety standard. ${ }^{10}$ Second, a large illumination area partially averages out the shadows of superficial heterogeneity in the image. Third, dark-field illumination reduces the otherwise strong interference of the extraneous photoacoustic signals from the superficial paraxial areas.

Photoacoustic signals received by the ultrasonic transducer are amplified by a low-noise amplifier (ZFL-500LN, Mini-Circuits) and recorded by a digital oscilloscope. The transducer is immersed in water inside a plastic container with an opening at the bottom that is sealed with a thin disposable polyethylene membrane. The sample (animal) is placed outside the container below the membrane, and the ultrasonic coupling is further secured by coupling gel.

Figure 2(a) shows four photoacoustic images of a Mylar U.S. Air Force Test Chart (USAF-1951) target taken through a 4-mm-thick layer of light-diffusing tissue phantom made from a $2 \%$ Intralipid solution (Clintec Nutrition Company, Dearfield, Illinois) and $1 \%$ agar gel. The estimated reduced scattering coefficient of the phantom, ${ }^{11} \mu_{s}{ }^{\prime} \approx 1.5 \mathrm{~mm}^{-1}$, is greater than that of most biological tissues. ${ }^{12}$ The thickness of the phantom translates into approximately six transport mean free paths. The numbers below the images in Fig. 2 indicate the spatial modulation frequency expressed in line pairs per millimeter. The solid curves show the relative ultrasonic pressure as a function of the horizontal displacement across the

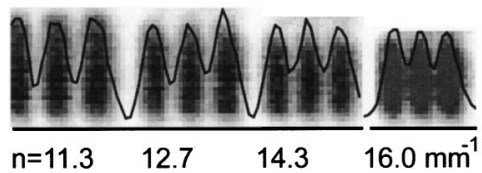

(a) (b)

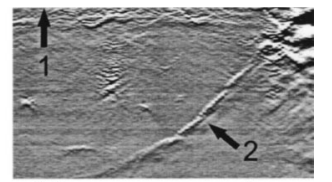

$\underline{1 \mathrm{~mm}}$
Fig. 2. (a) Image resolution test with a bar chart embedded $4 \mathrm{~mm}$ deep in a tissue phantom. (b) Imaging depth test with a black double-stranded cotton thread embedded obliquely in the abdominal area of a rat: 1 , skin surface; 2 , thread. bars on the target. The modulation transfer function ${ }^{13}$ was extracted from Fig. 2 and extrapolated to its cutoff spatial frequency, producing an estimated lateral resolution of $45 \mu \mathrm{m}$. The axial resolution was estimated to be $\sim 15 \mu \mathrm{m}$, based on the spread function of the photoacoustic signal from the top surface of an embedded object.

When a 1.2-mm-thick freshly harvested skin from a sacrificed rat was placed between the acoustic lens and the USAF-1951 target, the lateral resolution degraded to $\sim 120 \mu \mathrm{m}$, likely because of increased ultrasonic attenuation. Ultrasonic attenuation in the skin decreased the signal-to-noise ratio to $\sim 30 \mathrm{~dB}$ from $80 \mathrm{~dB}$ in clear water and to $\sim 50 \mathrm{~dB}$ in the phantom samples. Increasing the signal-to-noise ratio potentially may recover the resolution.

Figure 2(b) shows a photoacoustic B-scan (vertical cross section) image of a black double-stranded cotton thread of 0.2-mm diameter and 1.25-mm pitch, which was embedded obliquely in the abdominal area of a sacrificed rat. The thread is clearly visible in the image to a depth of $3 \mathrm{~mm}$, which shows the capability of our current system.

Data were collected on Sprague Dawley rats ( $\sim 180$ g; Charles River Breeding Laboratories). Before imaging, the hair on the backs of the rats was removed with commercial hair remover lotion. Imaging was performed under general anesthesia by intramuscular injection of ketamine hydrochloride $(44 \mathrm{mg} / \mathrm{kg})$, xylazine hydrochloride $(2.5 \mathrm{mg} / \mathrm{kg})$, acepromazine maleate $(0.75 \mathrm{mg} / \mathrm{kg})$, and atropine $(0.025 \mathrm{mg} / \mathrm{kg})$. During the procedure we maintained the animals' normal body temperature by controlling the immersion container, with additional heat provided as needed by an overhead surgical lamp. After several hours of experimentation, the rats recovered normally without noticeable health problems. Finally, the rats were sacrificed by use of pentobarbital $(120 \mathrm{mg} / \mathrm{kg}$, IP). The imaged skin was removed from the rats and photographed from the inner skin surface. All experimental animal procedures were carried out in compliance with the guidelines of the U.S. National Institutes of Health. ${ }^{14}$ The laboratory animal protocol for this work was approved by the University Laboratory Animal Care Committee of Texas A\&M University.

Figure 3 shows photoacoustic images of the vascular distribution in the dorsal dermis (the upper lumbar area left of the vertebra) of a Sprague Dawley rat. Four in situ consecutive photoacoustic B-scan images that were obtained $0.2 \mathrm{~mm}$ apart laterally are shown in Fig. 3(a). Each image is a gray-scale plot of the peak-to-peak amplitudes of the received photoacoustic signals; the vertical and horizontal axes represent the depth from the skin surface and the horizontal transducer position, respectively. We obtained the vertical axis by multiplying the acoustic time of arrival starting from the laser pulse by an assumed acoustic speed of $1500 \mathrm{~m} / \mathrm{s}$. The focal plane of the transducer was located at a depth of $1.2 \mathrm{~mm}$. The animal was scanned horizontally in $1000.1-\mathrm{mm}$ steps, which took $10 \mathrm{~s}$ to complete. The slightly in- 

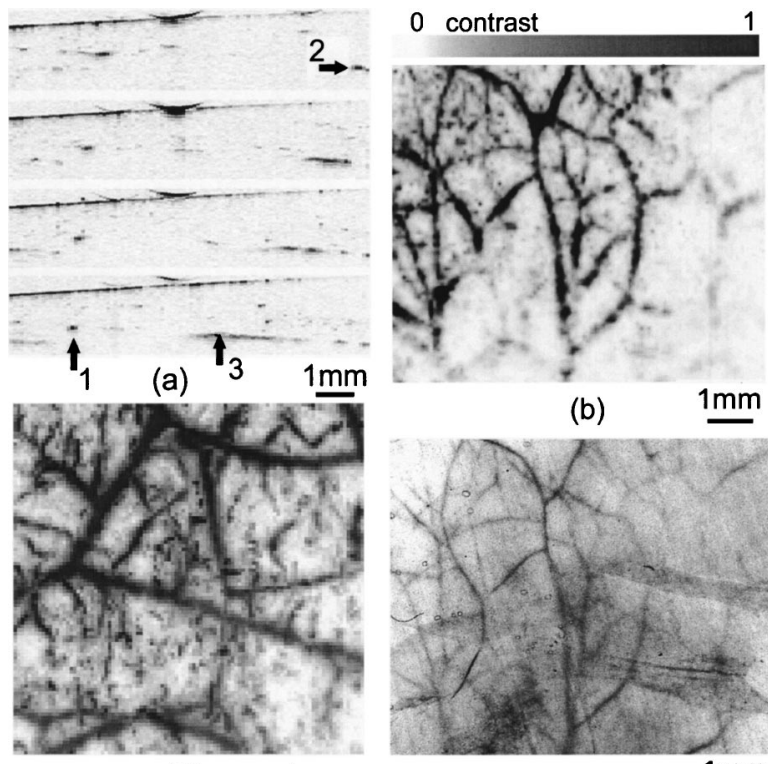

(d) $1 \mathrm{~mm}$

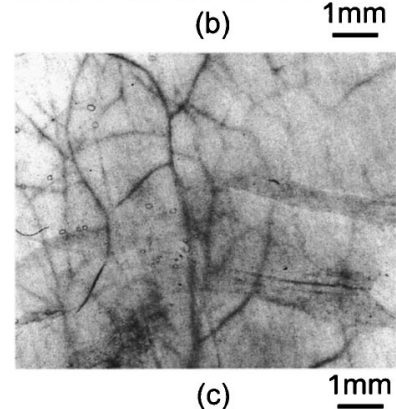

Fig. 3. Vascular distribution in rat skin. (a) Four in situ consecutive photoacoustic B scans: 1,2 , vessels perpendicular to the image plane; 3 , in-plane vessel. Comparison of (b) the in situ photoacoustic projection image taken from the epidermal side and (c) a photograph taken from the dermal side with transmission illumination. (d) In vivo noninvasive photoacoustic projection image taken from the epidermal side.

clined solid line in the upper part of each B scan delineates the skin surface. Some vessels, for example, the ones marked 1 and 2 in Fig. 3(a), are nearly perpendicular to the imaging plane of the B scans. Note that the size of vessel 1 is $\sim 0.1 \mathrm{~mm}$ ( 1 pixel) in the image, indicating that the resolution in the biological tissues is $\sim 0.1 \mathrm{~mm}$. Conversely, the vessel marked 3 in Fig. 3(a) is nearly parallel to the imaging plane.

Figure 3(b) shows an in situ photoacoustic projection image similar to a C-scan or en face image (100 $\times 100$ pixels; $0.1-\mathrm{mm}$ step size). The image is a grayscale plot of the maximum peak-to-peak amplitudes of the received photoacoustic signals within a $0.2-2-\mathrm{mm}$ depth interval from the skin surface versus the two-dimensional transducer position on the skin surface. For comparison, Fig. 3(c) shows a photograph of the inner surface of the harvested skin that was obtained with transmission illumination. Good agreement in the vascular anatomy can be observed between the photoacoustic image and the photograph. Based on the photograph [Fig. 3(c)], the major vessels are $\sim 100 \mu \mathrm{m}$ in diameter and the smaller vessels are $\sim 30 \mu \mathrm{m}$ in diameter.

Figure 3(d) shows an in vivo photoacoustic projection image of a similar area $(100 \times 100$ pixel, 0.05-mm step size, $0.5-3-\mathrm{mm}$ depth interval) taken in vivo. One can see an elaborate system of blood vessels with a density of as many as a few counts per millimeter. The signal-to-background ratio for the larger vessels is $\sim 40 \mathrm{~dB}$. A major problem for in vivo imaging is to overcome the motion artifact that results from the animal's breathing, which blurs the image.

To summarize, a dark-field confocal microscopic photoacoustic imaging technique has been developed to image biological tissues in vivo. The lateral resolution is as high as $45 \mu \mathrm{m}$ in tissue phantoms. The maximum imaging depth is at least $3 \mathrm{~mm}$. Further improvement of the image resolution by increasing the ultrasonic frequency is possible, at a cost of imaging depth. The photoacoustic images shown here were taken without signal averaging and therefore could be further improved by averaging, at the expense of data acquisition time. Our current imaging speed is limited by the pulse repetition rate of the laser that we used. Because lasers with pulse repetition rates of as much as $100 \mathrm{kHz}$ are now available, real-time photoacoustic imaging, which will reduce motion artifacts, is possible, and extensive signal averaging is also a realistic goal.

This project was sponsored by National Institutes of Health grants R01 EB000712 and R01 NS46214. L. V. Wang's e-mail address is lwang@tamu.edu.

\section{References}

1. H. Nakajima, T. Minabe, and N. Imanishi, Plast. Reconstr. Surg. 102, 748 (1998).

2. P. Carmeliet and R. K. Jain, Nature (London)407, 6801 (2000).

3. S. J. Nelson and S. Cha, Cancer J. Sci. Am. 9, 134 (2003).

4. X. Wang, Y. Pang, G. Ku, X. Xie, G. Stoica, and L. V. Wang, Nat. Biotechnol. 21, 803 (2003).

5. M. Xu and L. V. Wang, IEEE Trans. Biomed. Eng. 50, 1086 (2002).

6. A. A. Oraevsky, V. A. Andreev, A. A. Karabutov, D. R. Fleming, Z. Gatalica, H. Singh, and R. O. Esenaliev, Proc. SPIE 3597, 352 (1999).

7. C. G. A. Hoelen, F. F. M. de Mul, R. Pongers, and A. Dekker, Opt. Lett. 23, 648 (1998).

8. R. G. M. Kolkman, E. Hondebrink, W. Steenbergen, and F. F. M. de Mul, IEEE J. Sel. Top. Quantum Electron. 9, 343 (2003).

9. C. Guittet, F. Ossant, L. Vaillant, and M. Berson, IEEE Trans. Biomed. Eng. 46, 740 (1999).

10. American National Standards Institute, "American National Standard for the Safe Use of Lasers," ANSI Standard Z136.1 (American National Standards Institute, New York, 2000).

11. S. T. Flock, S. L. Jacques, B. C. Wilson, W. M. Star, and M. J. C. van Gemert, Lasers Surg. Med. 12, 510 (1992).

12. W. F. Cheong, S. A. Prahl, and A. J. Welch, IEEE J. Quantum Electron. 26, 2166 (1990).

13. W. J. Smith, Modern Optical Engineering, (McGrawHill, New York, 1966), p. 318.

14. National Institutes of Health, "Guide for the care and use of laboratory animals, revised ed.," NIH Publ. 86-23 (U.S. Government Printing Office, Washington, D.C., 1985). 